\title{
النظريات المفسرة لصعوبات التعلم وتطبيقاتها التربوية في غرفة الصف
}

\author{
Théories explicatives des difficultés d'apprentissage et leurs applications \\ pédagogiques en classe
}

تاريخ الاستلام : 2019/08/15 ؛ تاريخ القبول : 2020/11/17

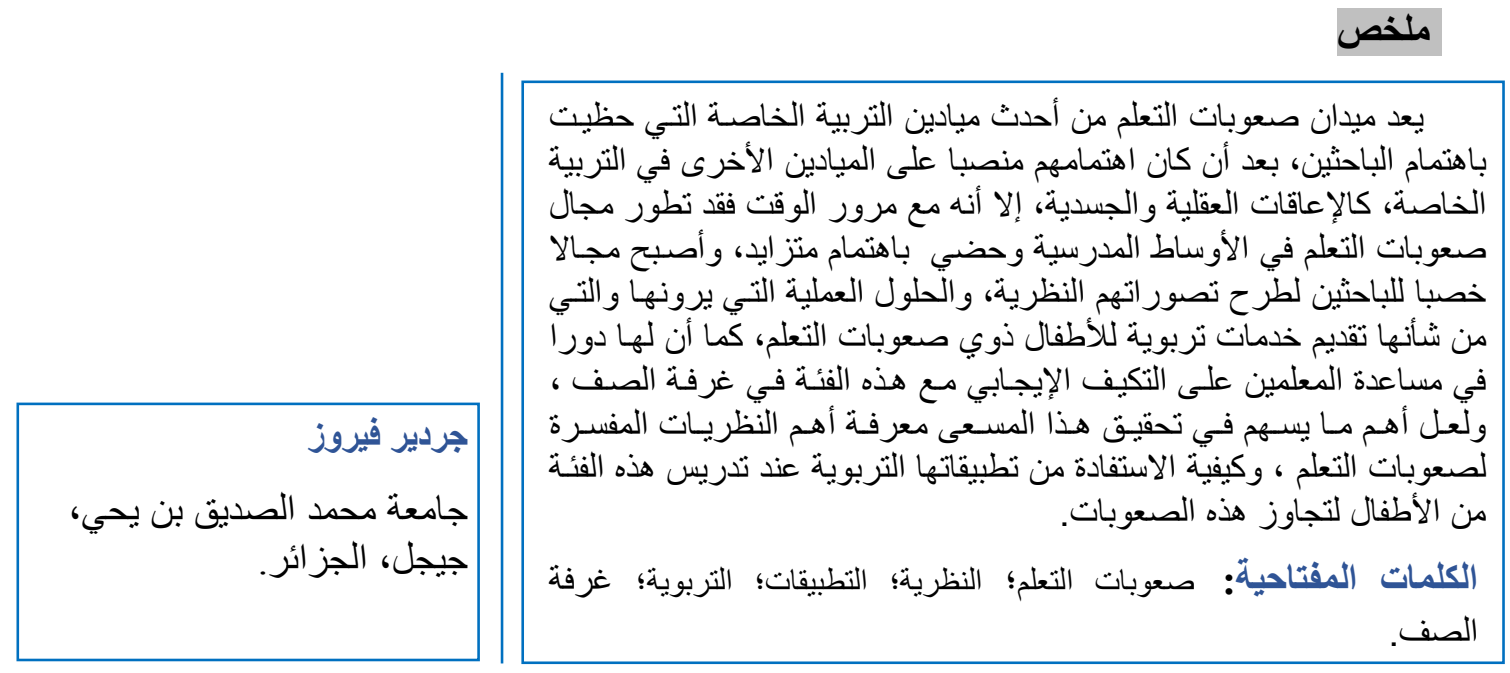

\section{Abstract}

The field of learning difficulties is one of the most recent fields of special education that has attracted the attention of researchers, after their attention was focused on other fields in special education, such as mental and physical disabilities, but with the passage of time the field of learning difficulties has evolved in the school community and has received increasing attention, and has become a fertile field For researchers to present their theoretical perceptions, and the practical solutions that they see that would provide educational services for children with learning difficulties, as it has a role in helping teachers to adapt positively with this group in the classroom, and perhaps the most important contribution to achieving this goal is to know the most important theories Navel learning difficulties, and how to take advantage of educational applications when teaching this group of children to overcome these difficulties

Keywords: learning disabilities; theory; educational 1; applications; classroom .

\section{Résumé}

Le domaine des difficultés d'apprentissage est l'un des domaines les plus récents de l'éducation spécialisée qui a attiré l'attention des chercheurs, après que leur attention ait été concentrée sur d'autres domaines de l'éducation spéciale, tels que les handicaps mentaux et physiques, mais avec le temps, le domaine des difficultés d'apprentissage s'est développé dans la communauté scolaire et a reçu une attention croissante et est devenu un domaine fertile. Pour les chercheurs, afin de présenter leurs perceptions théoriques et les solutions pratiques qui, selon eux, fourniraient des services éducatifs aux enfants ayant des difficultés d'apprentissage, car cela a un rôle à jouer pour aider les enseignants à s'adapter positivement avec ce groupe en classe, et la chose la plus importante qui contribue à cet effort est de connaître les théories les plus importantes des difficultés d'apprentissage Interprété, et comment tirer profit des applications pédagogiques pour enseigner ce groupe d'enfants à surmonter ces difficultés.

Mots clés: troubles d'apprentissag ; théorie; applications ; éducatives ; Salle de classe;

Corresponding author's e-mail: fay.rose81@yahoo.fr 


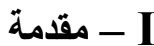

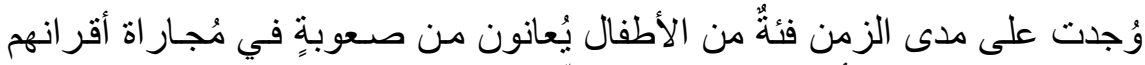

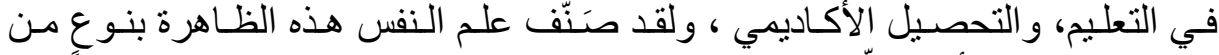

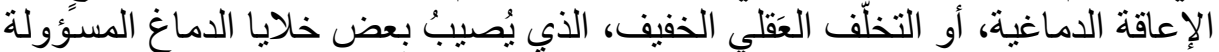

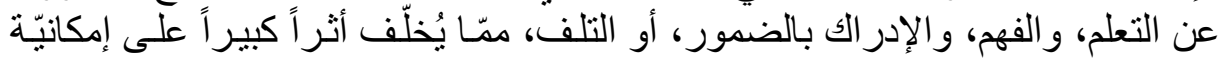

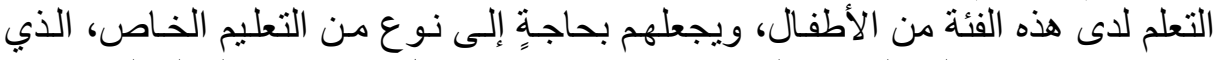

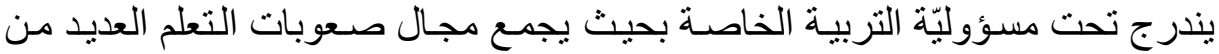

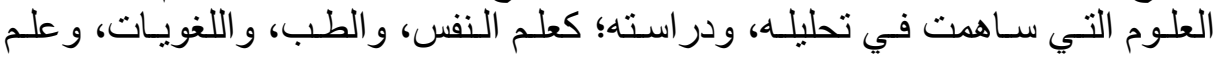

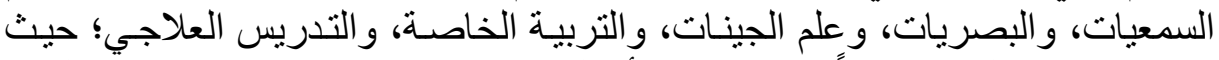

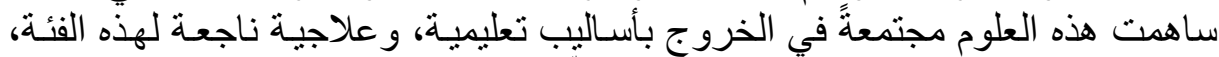

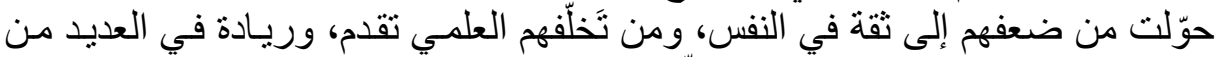

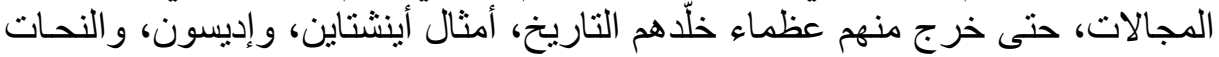

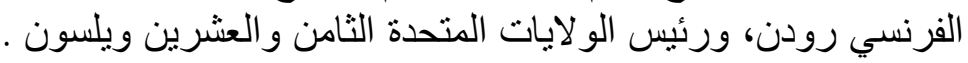

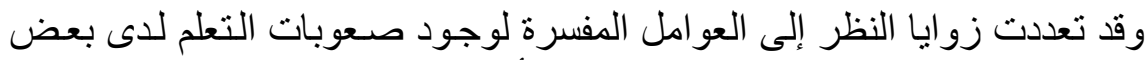

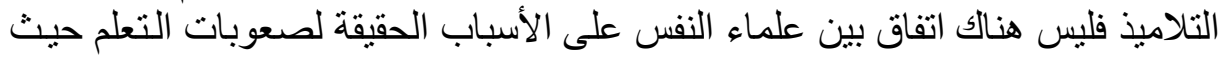

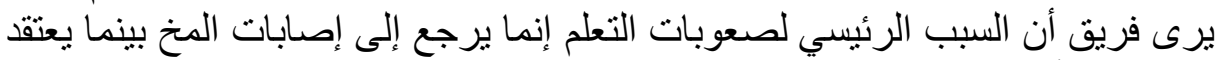

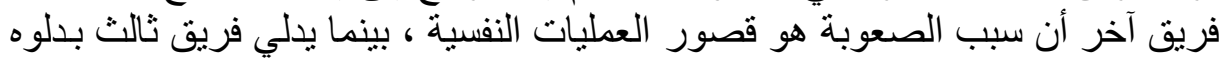

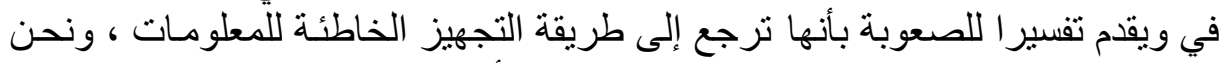

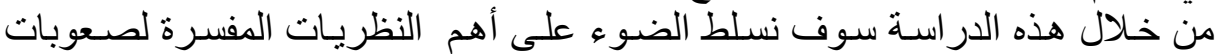
التعلم وكيفية الاستفادة من تطبيقاتها التربوية عند تدريس هذه الفئة من الأطفال لتجاوز

هذه الصعوبات.

الإثكالية: - الإه

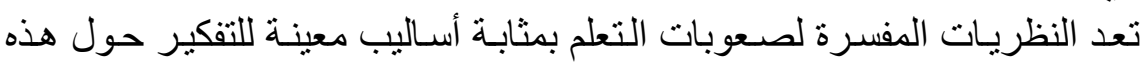

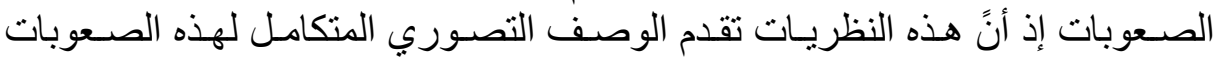

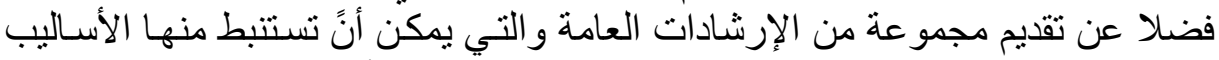

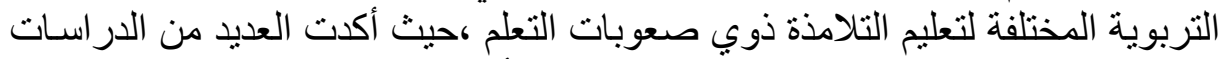

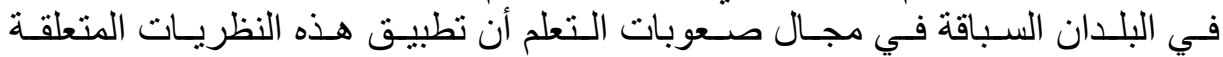

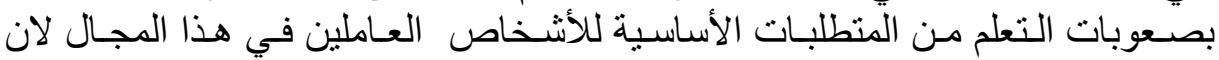

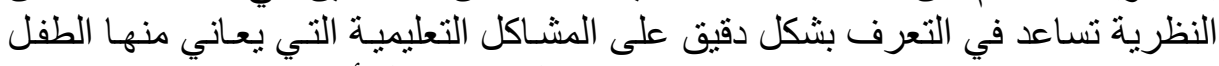

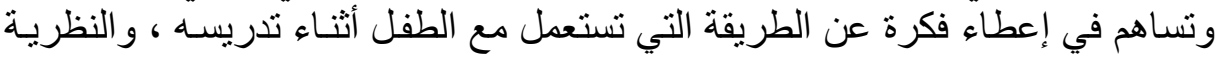

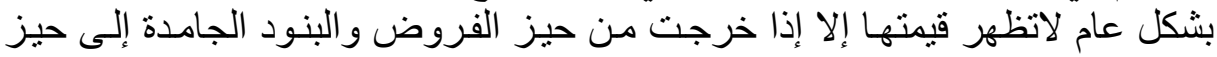

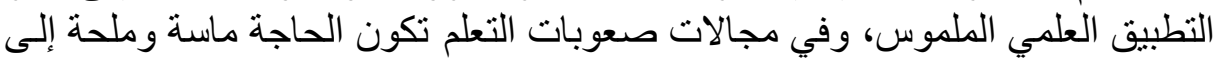

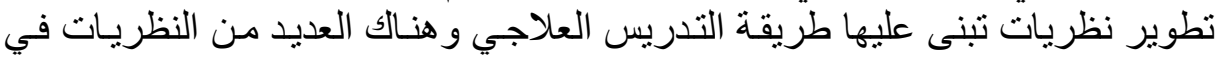

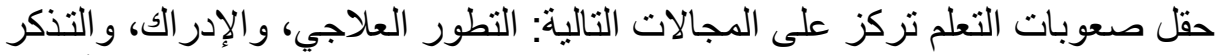

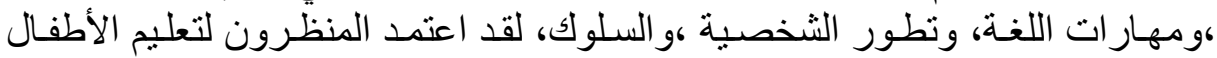

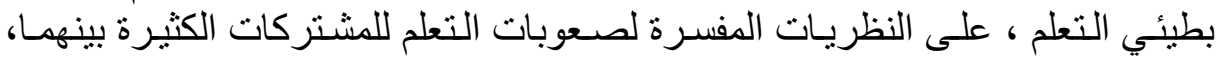

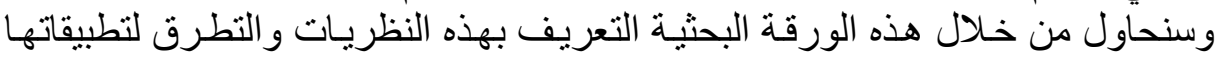
التربوية داخل غرفة الصف ، وذلك من خلال الإجابة على التساؤلات التالية:

$$
\begin{aligned}
& \text { • ما هي الأسس و المبادئ التي ترتكز عليها هذه النظريات ؟ } \\
& \text { • فيما تتمثل تطبيقاتها التربويةّ ؟ }
\end{aligned}
$$




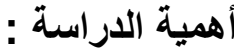

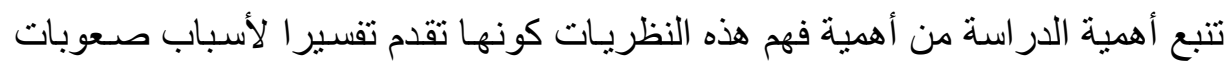

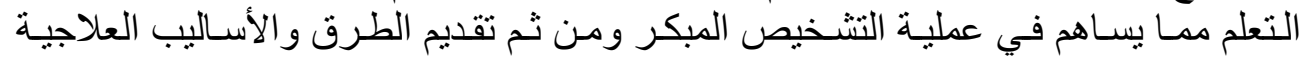

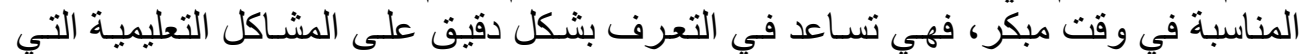

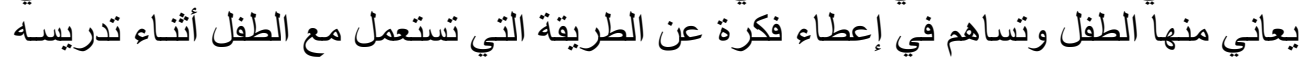

$$
\begin{aligned}
& \text { أهداف الدراسة: تتمثل أهداف الدراسة في: } \\
& \text { • التعرف على الأسس والمبادئ التي ترنكز عليها هذه النظريات؛ }
\end{aligned}
$$

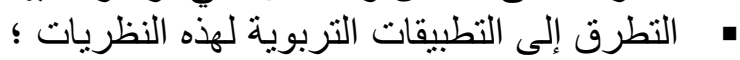

لغة : إن مفهوم النظرية:

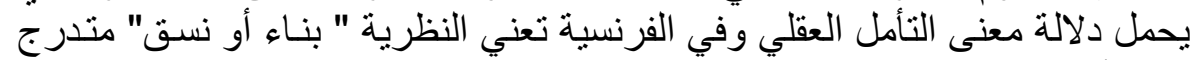

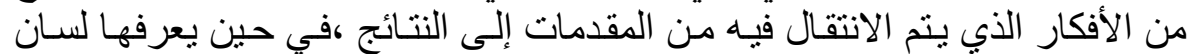

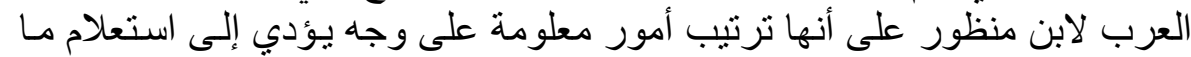

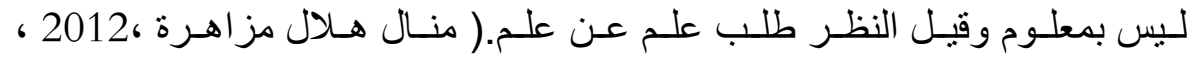

ص168)

اصطلاحا: النظرية عبارة عن مجموعة من المفاهيم و التعريفات والاقتر احسات التي

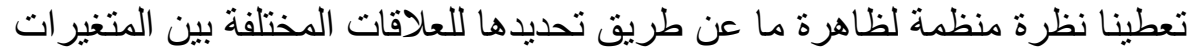

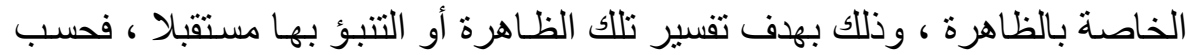

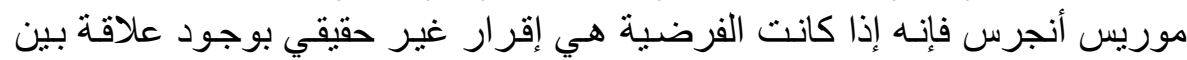

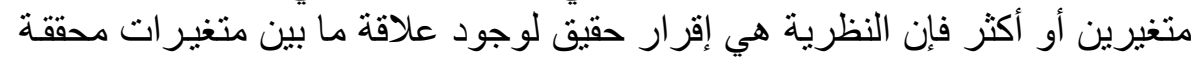

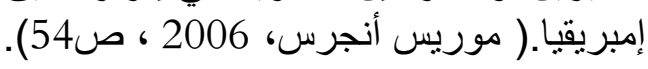

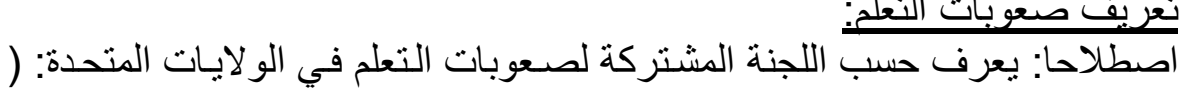

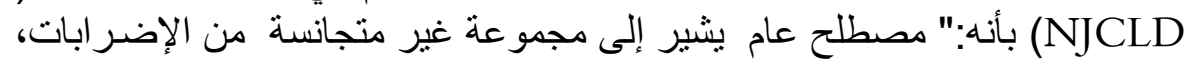

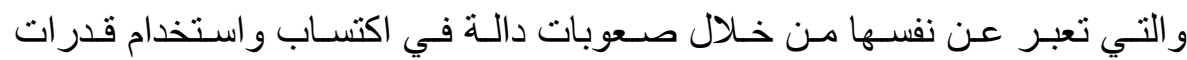

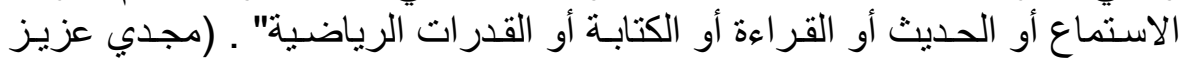

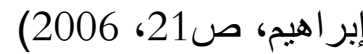

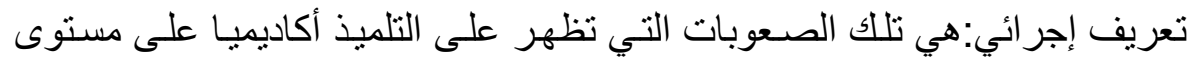

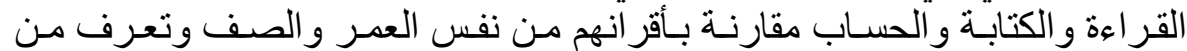
خلال نتائج التلميذ الدراسية. تعريف التطبيقات التربوية:

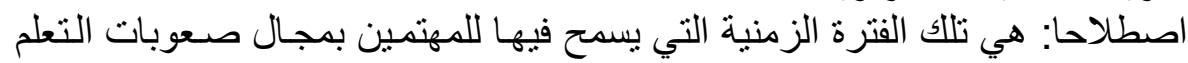

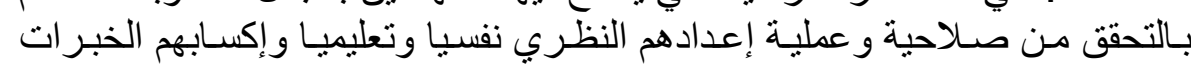

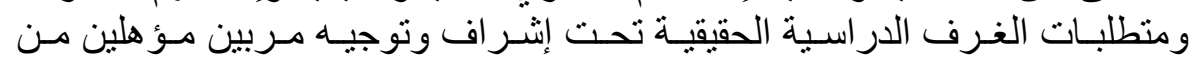

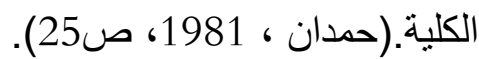
تعريف إجرائي: هي مرحلـة هامـة وضـرورية من من مر احل إعداد المعلمين الأكفاء

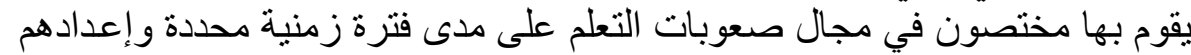

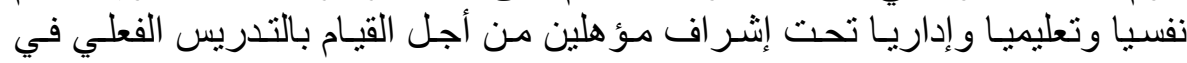

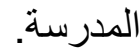




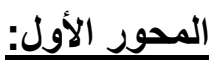

النظريات المفسرة لصعوبات التعلم:

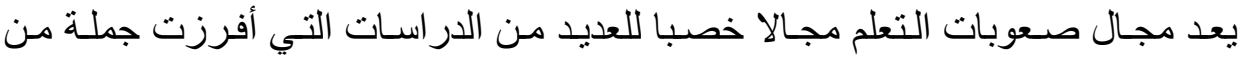

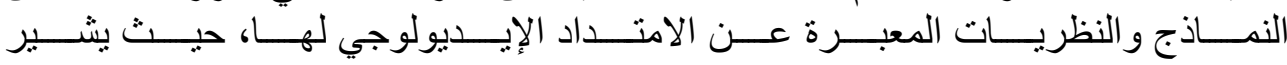

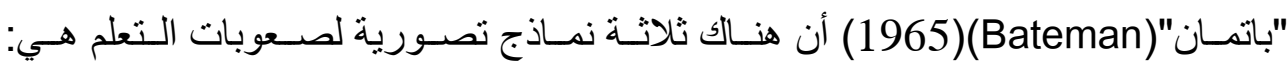

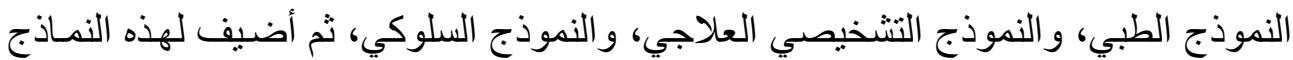

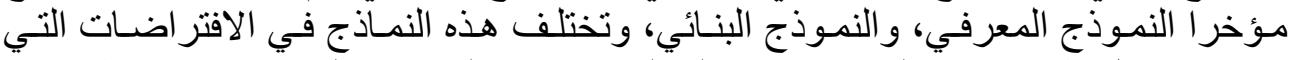

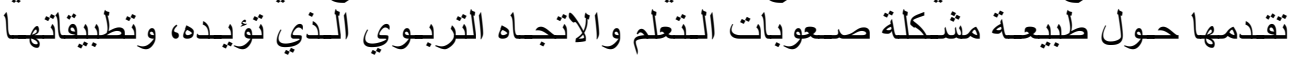

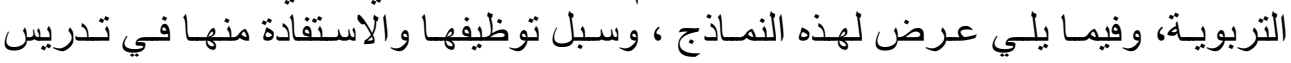

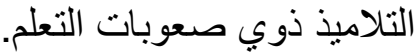

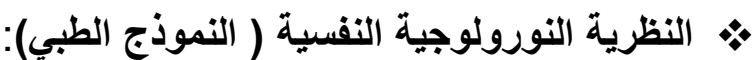

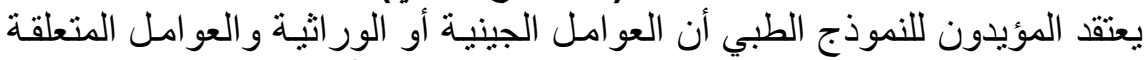

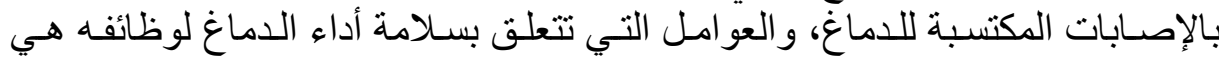

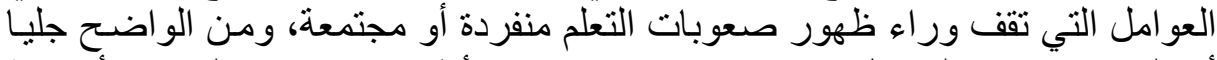

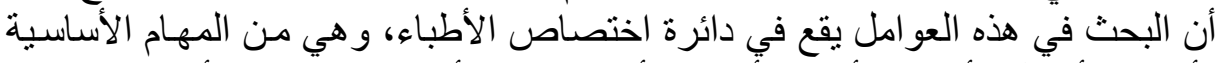

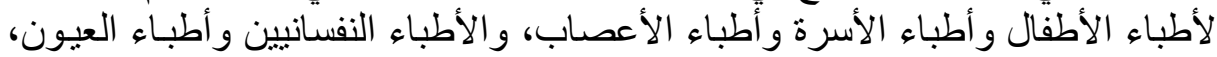

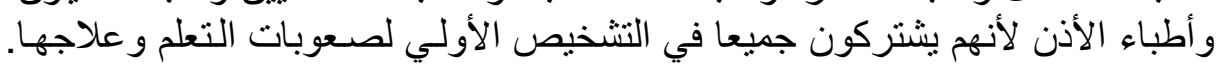

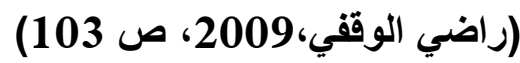

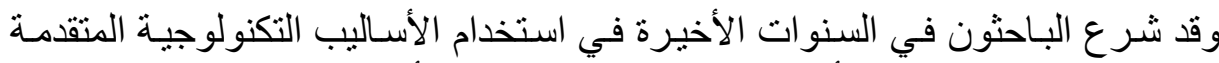

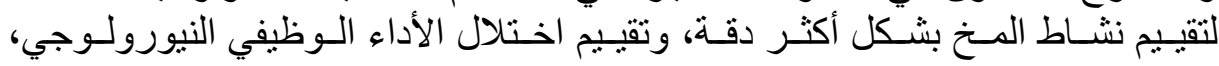

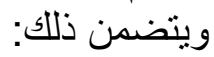

$$
\begin{aligned}
& \text { الأثعة المقطعية على المخ (CAT). } \\
& \text { ل أنشعة الرنين المغناطيسي (MRI) } \\
& \text { ل أنثعة الرنين المغناطيسي الوظيفي(IRMF) }
\end{aligned}
$$

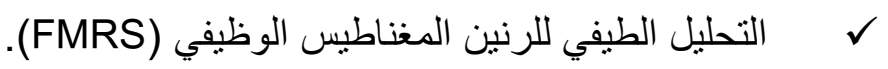

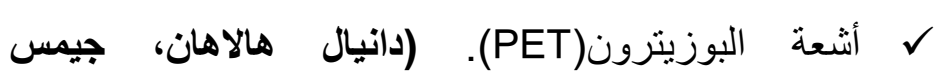

\section{كوفمان، 2008، 325 (ل)}

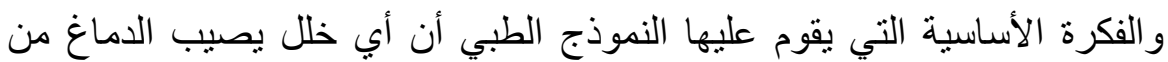

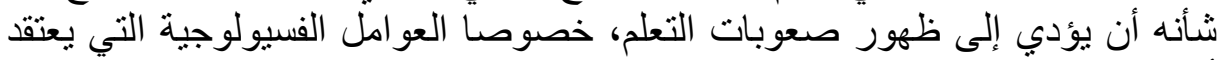

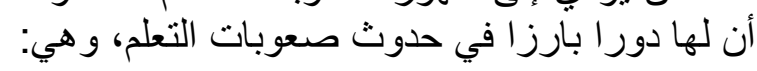

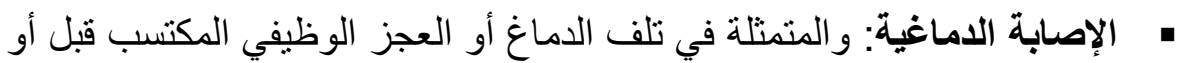

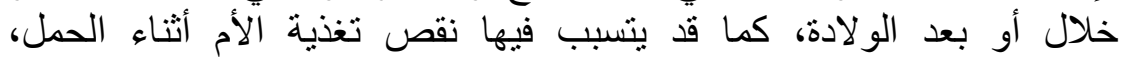

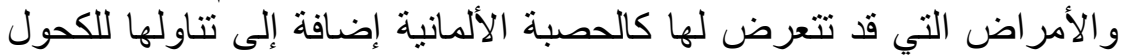

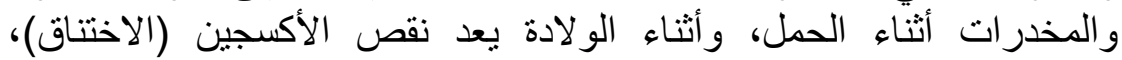

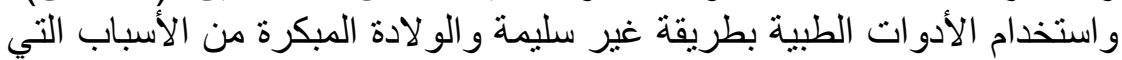

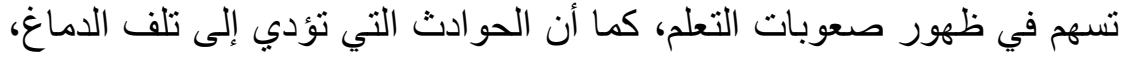


كالتهاب الدماغ، و التهاب السحايا، و الحصبة الألمانية، و الحمى القرمزية بمكن إلفابن

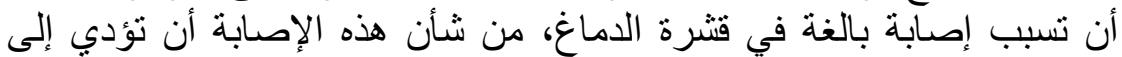

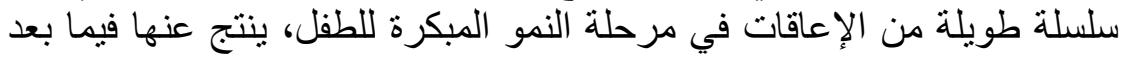

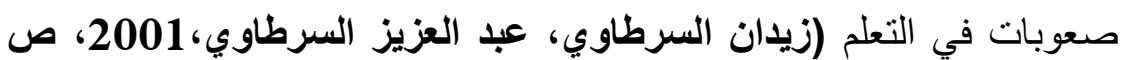

كما تذهب عدة دراسات إلى دور الوراثة في الإصابة بصعوبات التعلم، فقد

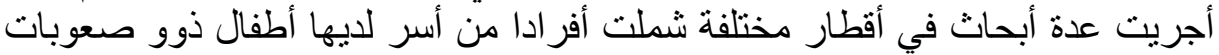

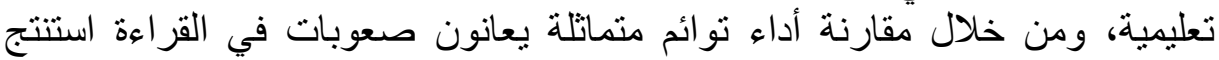

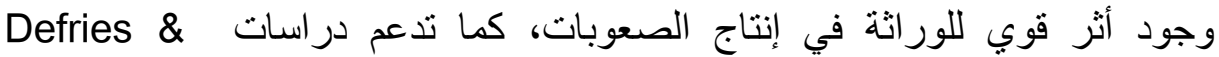
Decker

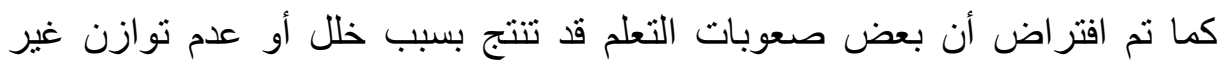

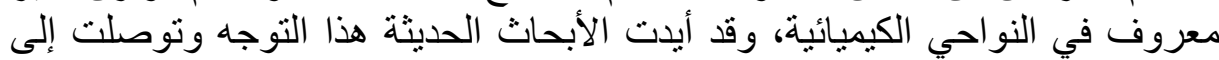

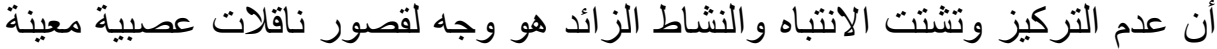

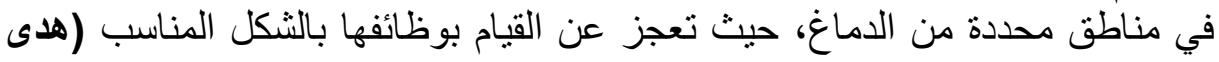

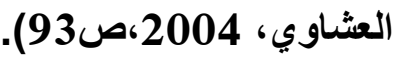

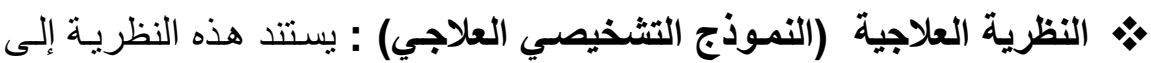

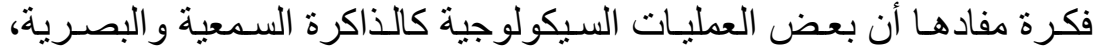

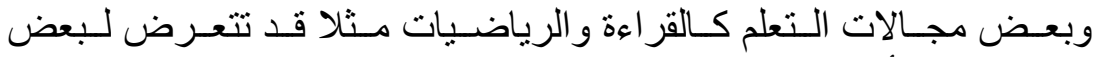

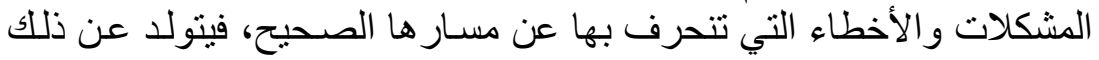

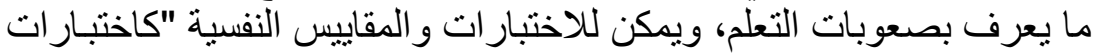

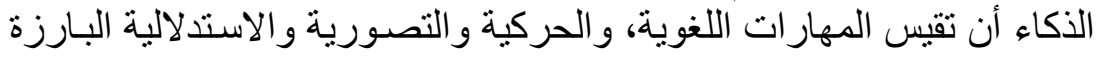

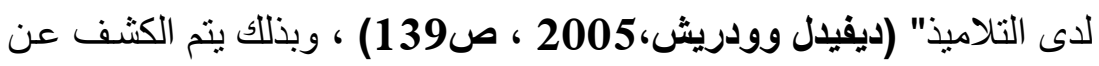

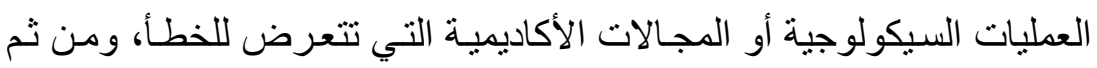

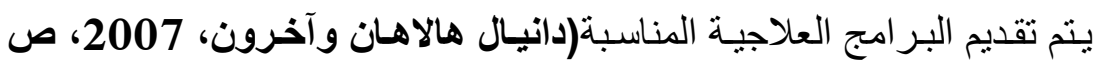

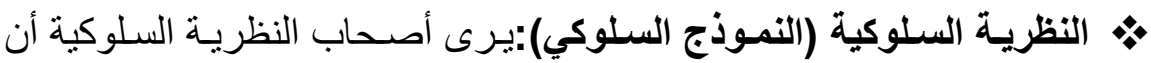

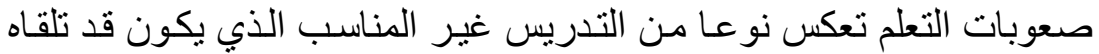

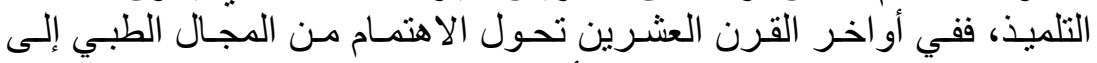
المجال التربوي نتيجة لثناثة عو امل أولية الفئن

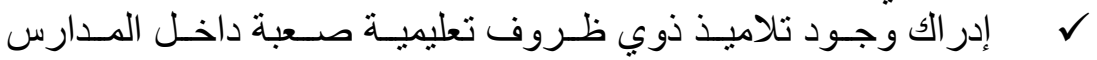

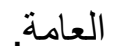
$\checkmark$ عدم وجود شـوا هد و أدلة كافية على قدرة الفحوص الطبيـة على التمييز

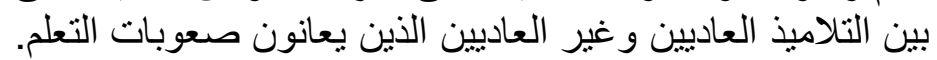

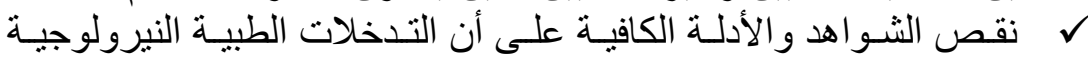

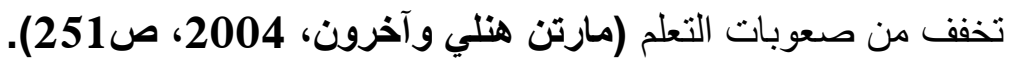

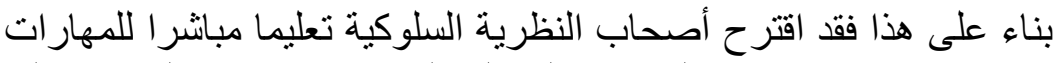

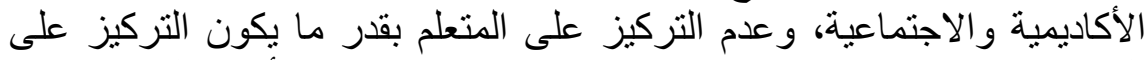

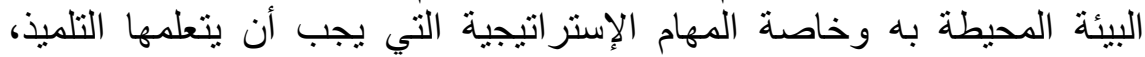


ولذلك يؤكد السلوكيون على العلاج المباثر والصريح للمشكلات الأكثر وضوحا

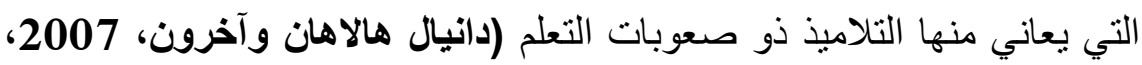

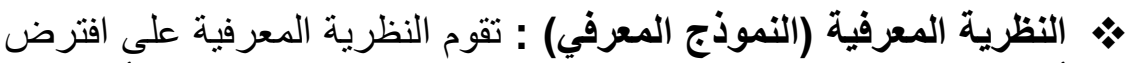

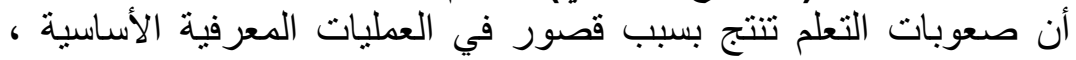

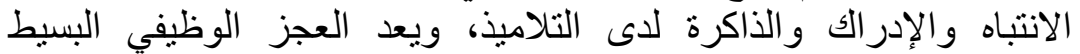

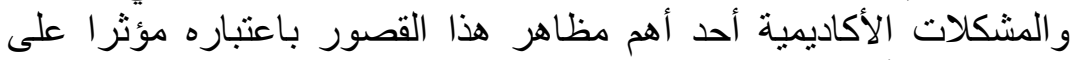

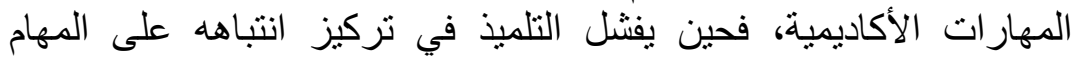

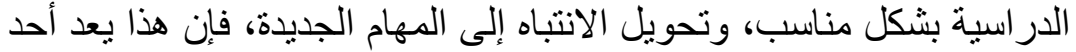

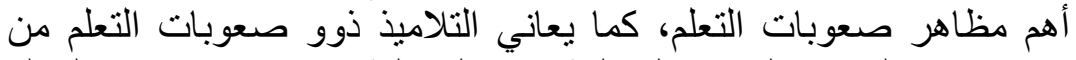

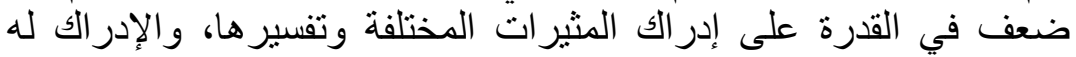

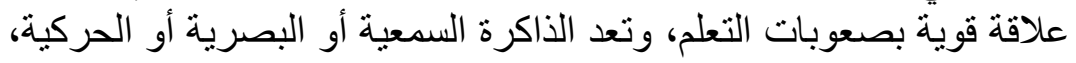

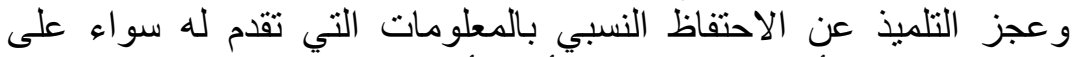

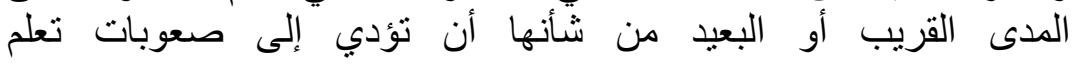

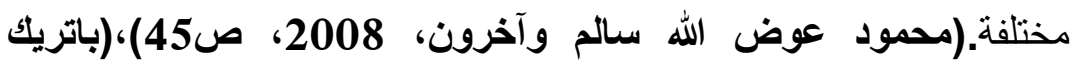

• النظرية البنائية(النموذج البنائي): تقوم النظرية البنائية على فكرة مفادها

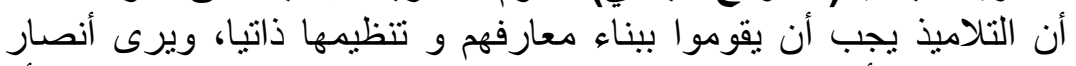

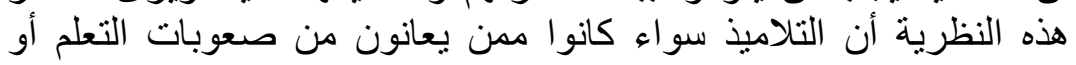

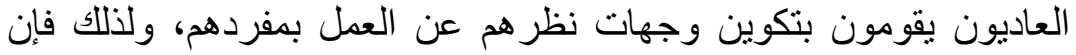

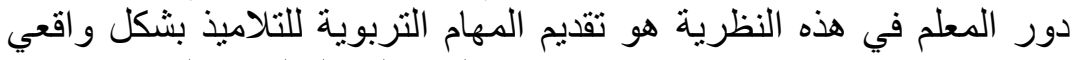

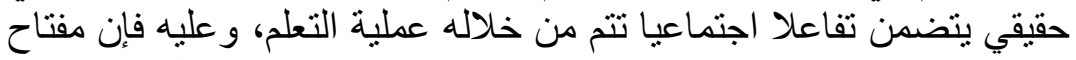

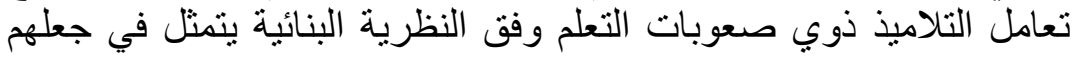

يقومون ببناء معارفهم خلال المو اقف الاجتماعية الحقيقية.

المحور الثانى:

التطبيقات التربوية للنظريات المفسرة لصعوبات التعلم وأهم التتخلات العلاجية. 1- 1 التطبيقات التربوية:

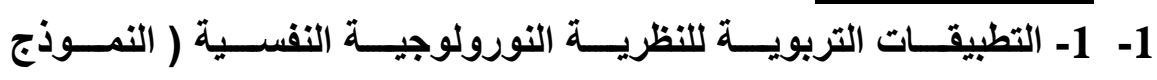

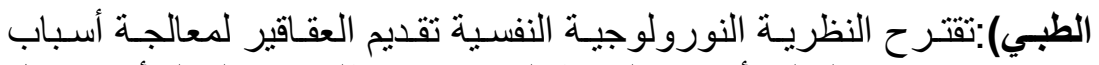

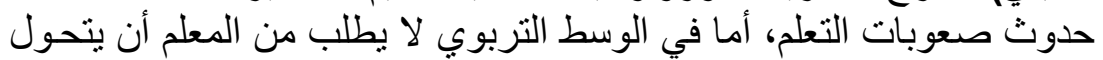

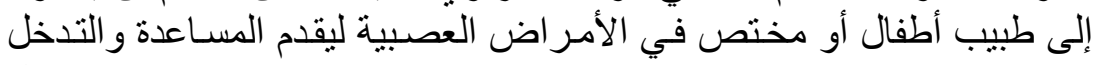

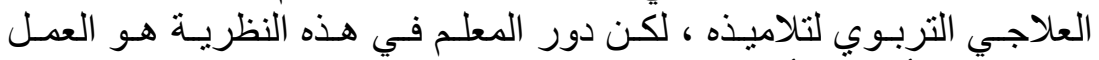

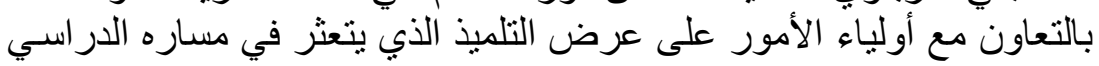

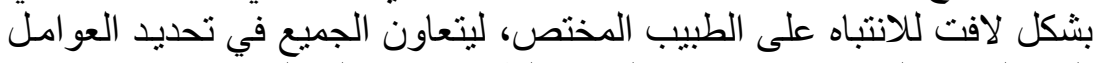

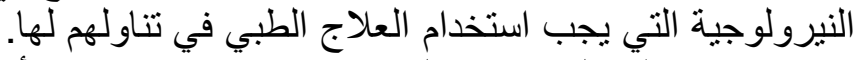

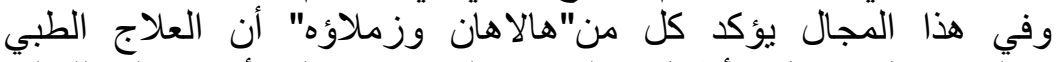

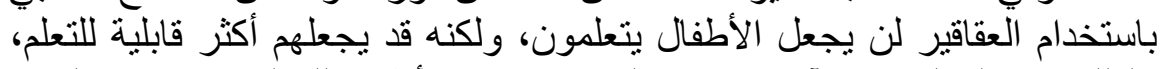

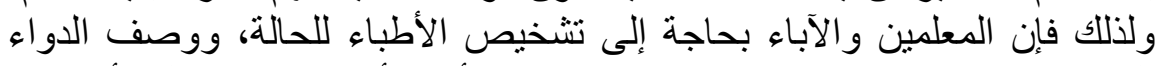
المناسب ومقدار الجرعة عند الضرورة، كما أن الأطباء بحاجة إلى لـاء أن يقوم 
المعلمون والآباء بتقديم التغذية الراجعة الدقيقة حول التعلم الأكاديمي للتلميذ

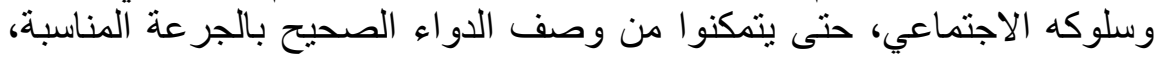

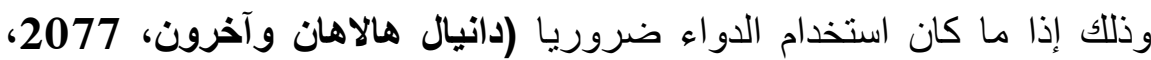

ص437).

1- 2- التطبيقات التربوية للنظرية العلاجية (النموذج التشخيصي العلاجي): تقترح

النظرية العلاجية في المجال التربوي استخدام الاختبار ات المعيارية التي تقدم لنابه

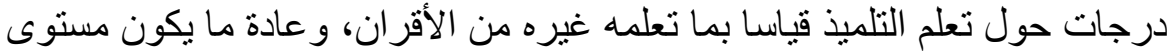

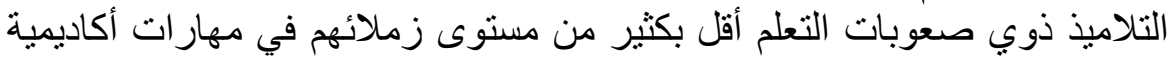

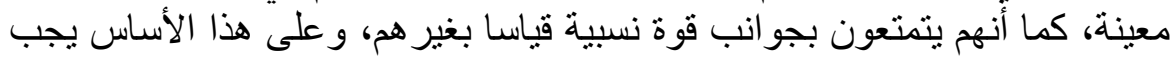

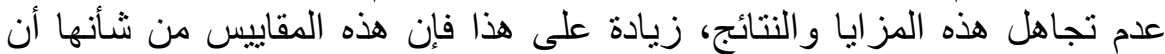
تساعد المعلمين في تضييق مجالات الاحتمالات عندما يتعلق الأمر بتحديد حاجيات

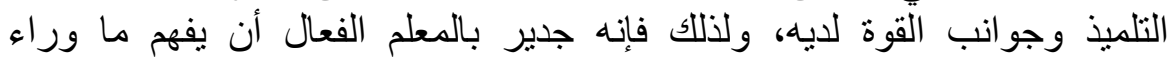

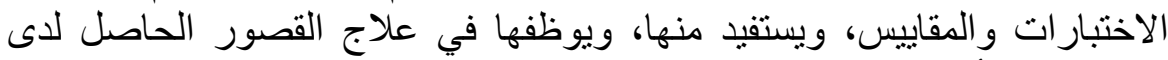

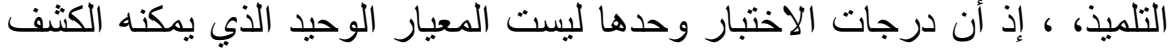

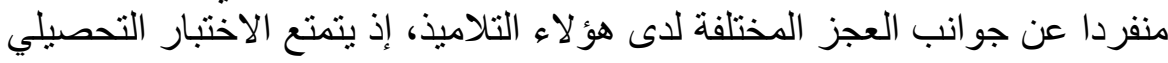

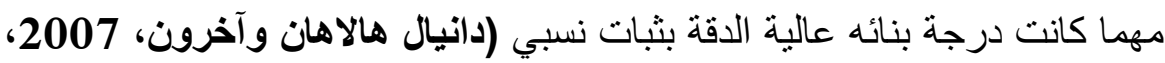

ص437). (437)

1- 3- التطبيقات التربوية للنظرية السلوكية (النموذج السلوكي): تقترح النظرية

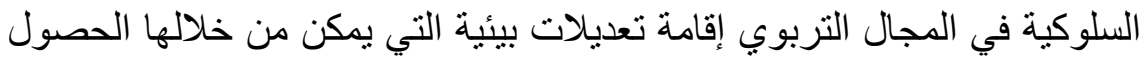

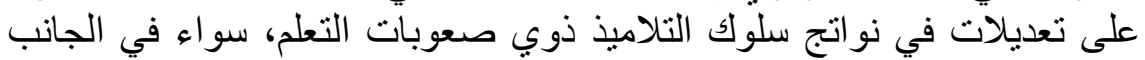

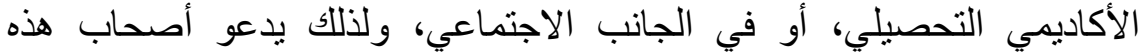

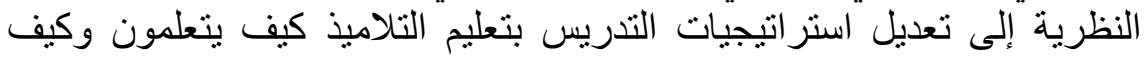

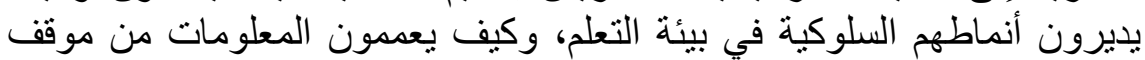

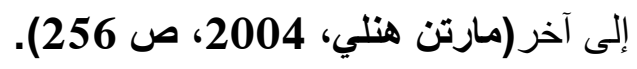

كما يؤكد السلوكيون على استخدام المعلمين للعديد من الأدوات المفيدة لأداء

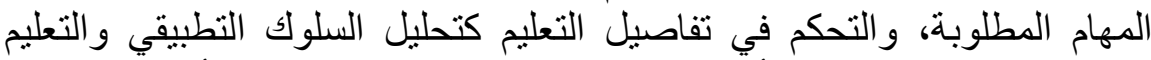

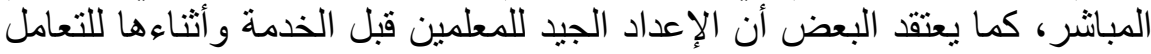

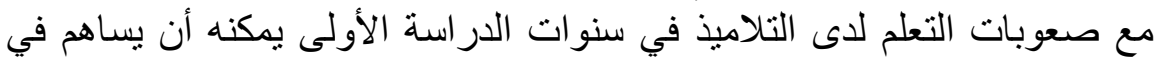

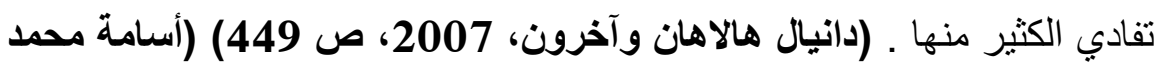

$$
\text { البطاينة وآخرون، 2009، ص 195). }
$$

\section{1 -4- التطبيقات التربوية للنظرية المعرفية (النموذج المعرفي):}

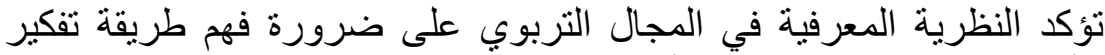

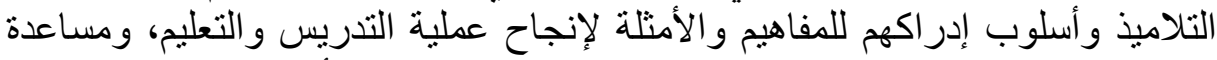

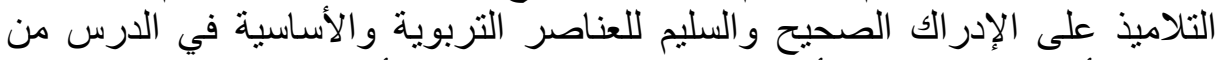

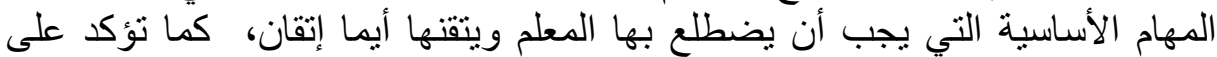

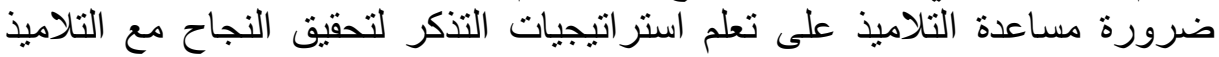

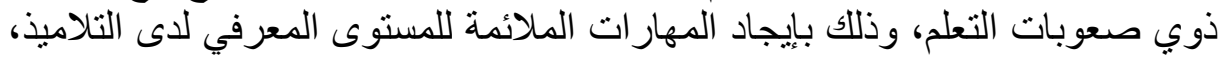

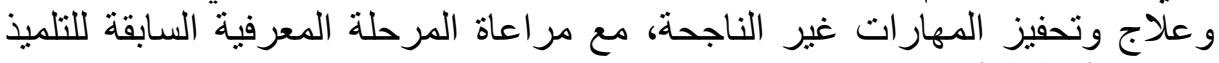


إلى جانب ما سبق تُعدّ تنمية الوعي الذاتي والحديث الذاتي والضبط الذيّ الذاتي

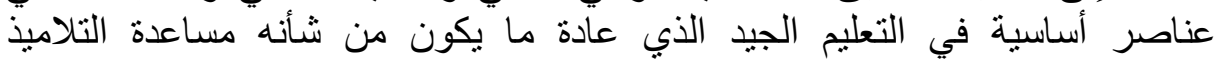
المصابين بصعوبات التعلم على حل المشكلات المبلد المدرسية اليومية، أو مشكلات مانه الحياة بوجه عام (محمود عوض الله سالم وآخرون، 2008، ص 48)؛(دانيال هالاهان

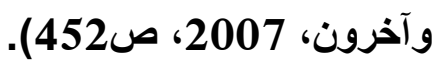

1 -5 - التطبيقات التربوية للنظرية البنائية (النموذج البنائي ): تقترح النظرية البنائية في المجال التربوي توظيف الأساليب الاجتماعية في التعلم، وتحقيق التفاعل الاجتماعي بين المعلم وتلاميذه، وبين التلاميذ أنفسهم لما لذلك من فاعلية في تحقيق

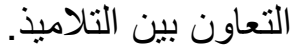

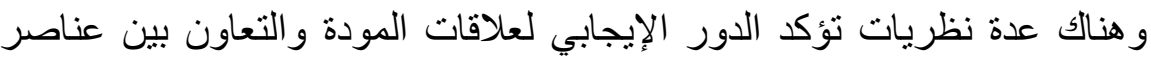

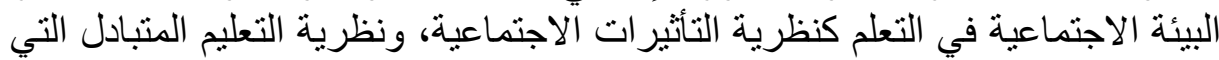

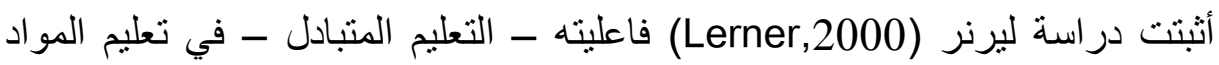

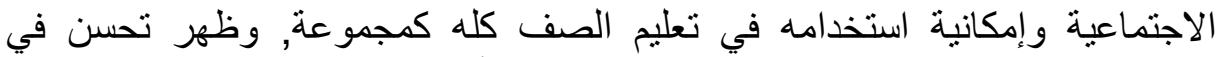

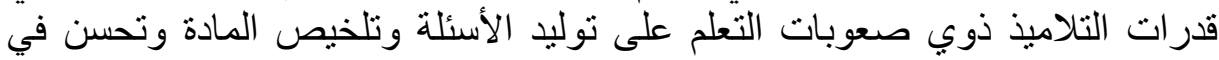
تفاعلهم واشتر اكهم في النقاش في المجموعات الحوارية ـ (راضي الوقفي، 2009،

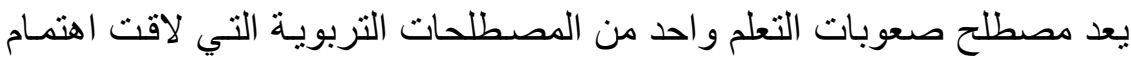

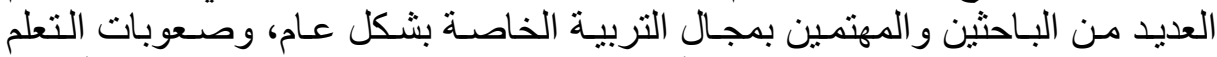

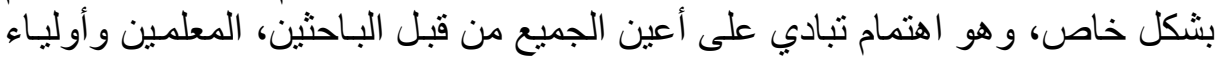

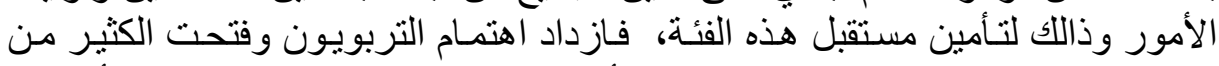

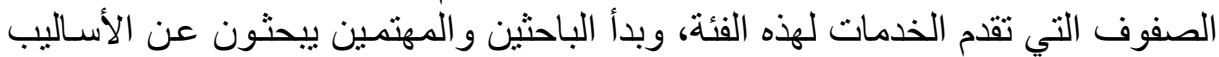

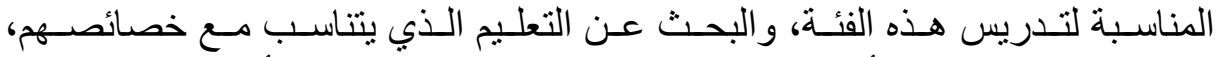

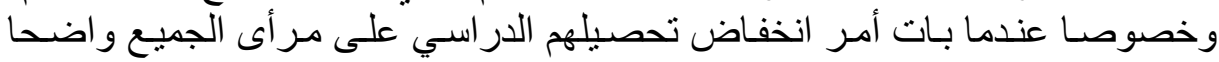

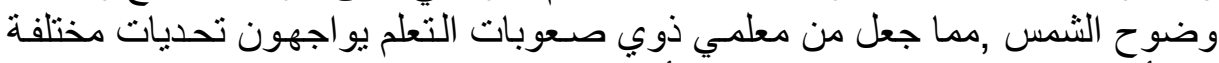

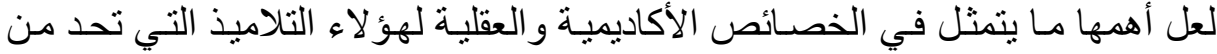

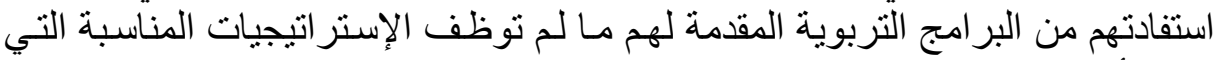

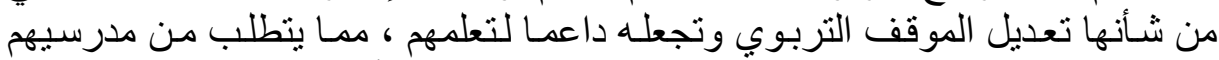

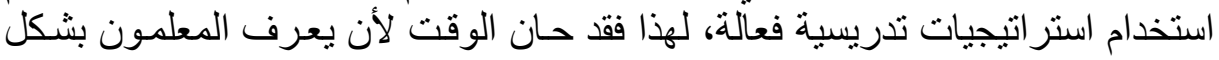

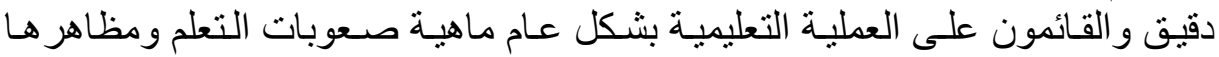

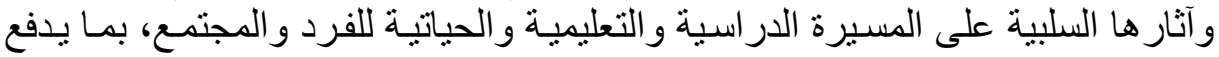

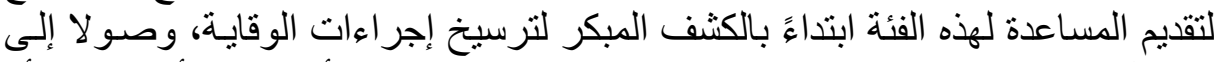

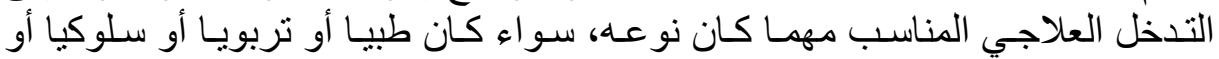

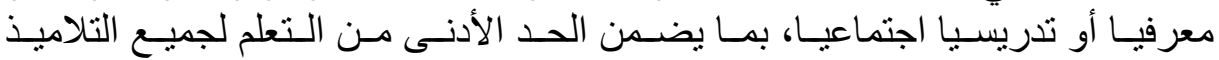

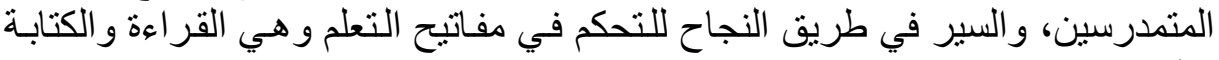

عدم الاكتفاء و الاعتماد كلية على حصص المعالجة كوسيلة للتكفل بذوي صعوبات التعلم

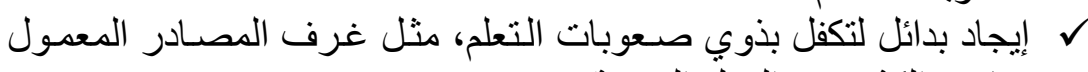
بها في الكثير من الدول العربية. 
ل الثرَكيز أكثر أثتاء تكوين المعلمين على طرق التشخيص و التشخيص

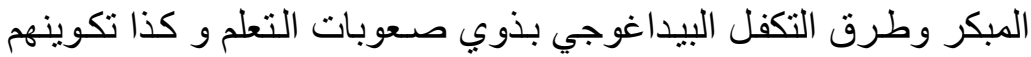

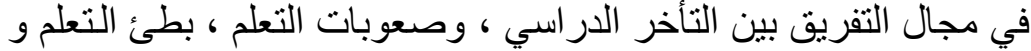

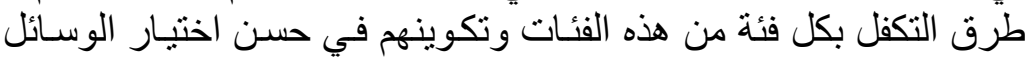

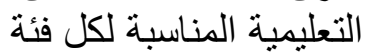

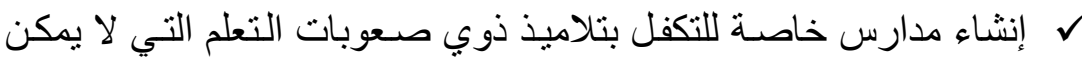
التكفل بها في المدارس العادية.

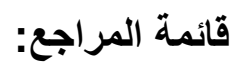

1. أسامة محمد البطاينة وَعبد الناصر ذياب الجراح وَمأمون محمود غوانمة

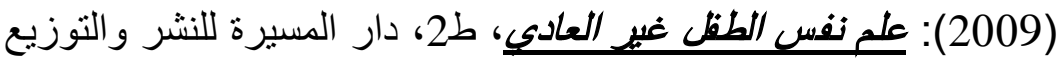

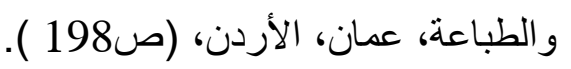

2. باتريك لومير، نرجمة عبد الكريم غريب (2011): علم النفس المعرفي

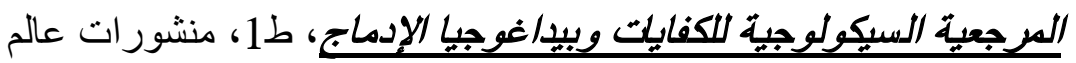

$$
\text { التربية, الدار البيضاء, المغرب ،( ص 31). }
$$

3. حمدان محمد زياد، (1981): التربية العلمية الميد/نية مفاهيهها، كفاياتهاه

$$
\text { معارساتها، الدار الحديثة، عمان، الأردن ، (ص25). }
$$

4. دانيال ـ ب. هالاهان وَ جيمس. م. كوفمان، ترجمة عادل عبد الله محمد

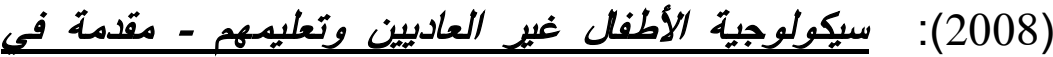

التربية الخلصة، ط1، دار الفكر ناتشرون وموزعون، عمان، الأردن،

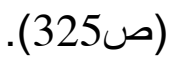

5. ديفيدل. وودريش، ترجمة كريمان بدير (2005): القياس النفسي للأطفال

دليل غير الأخصائي النفسي، ط1، عالم الكتب، القاهرة، مصر،

$$
\text { (ص) (139) (139) }
$$

6. راضي الوقفي (2004): أساسيت التربية الخلهة، ب ط، جهينة للنشر

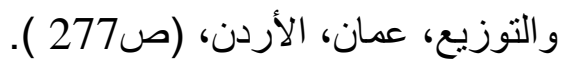

7. راضي الوقفي (2009): صعوبات التعلم النظري والتطبيقي، ط1، دار

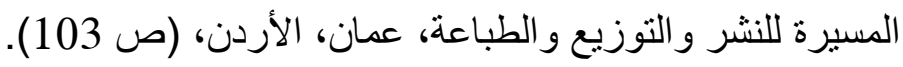

8. زيدان أحمد السرطاوي وَ عبد العزيز مصطفى السرطاوي، وَ أيمن إبراهيم

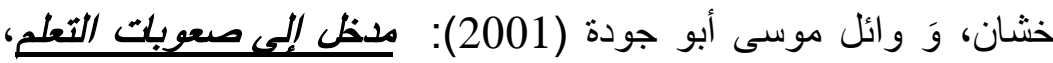

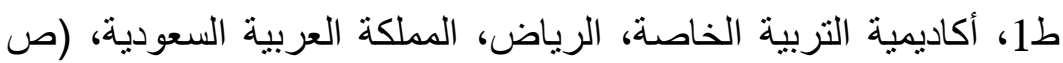


9. مارتن هنلي وَر وبرتا رامزي ألجوزين، ترجمة جابر عبد الحمبد جابر

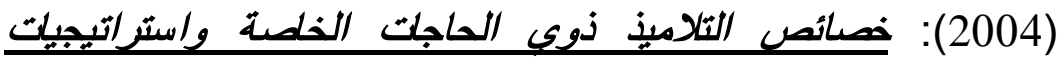

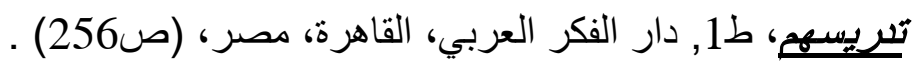
10. مجدي عزيز إبراهيم (2006): موسوعة المعارف الثربوية،، ط1، عالم

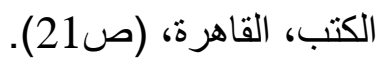
11. محمود عوض الله سالم ، وَ مجدي أحمد الثحات، وَ أحمد حسن عاثور

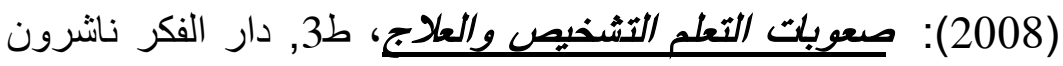

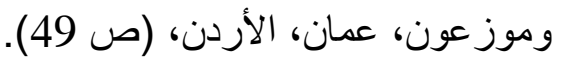

12. منال هلال مزاهرة (2012): نظريات الاتصل، دار المسيرة، الأردن ،

$$
\text { (ص) }
$$

13. موريس أنجرس(2006) : منهجية البحث العلمى في العلوم الإنسانية العية

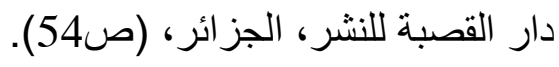

14. هدى عبد الله الحاج عبد الله العشاوي (2004): أطفالنا وصعوبات التعلم

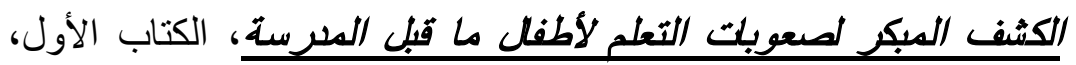

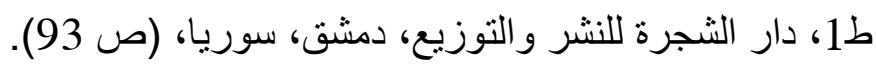

